\title{
The Hamburg/SAO survey for emission-line galaxies
}

\section{The third list of 81 galaxies}

\author{
U. Hopp ${ }^{1,9}$, D. Engels ${ }^{2}$, R.F. Green ${ }^{3}$, A.V. Ugryumov ${ }^{4}$, Y.I. Izotov $^{5,10}$, H.-J. Hagen ${ }^{2}$, A.Y. Kniazev ${ }^{4}$,

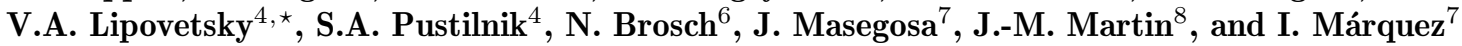 \\ 1 Universitätssternwarte München, Scheiner Str. 1, D-81679 München, Germany \\ 2 Hamburger Sternwarte, Gojenbergsweg 112, D-21029 Hamburg, Germany \\ 3 National Optical Astronomy Observatories, Tucson, AZ 85726-6732, U.S.A. \\ 4 Special Astrophysical Observatory, Nizhnij Arkhyz, Karachai-Circessia 357147, Russia \\ 5 Main Astronomical Observatory, Goloseevo, Kiev-127 03680, Ukraine \\ 6 Wise Observatory, Tel-Aviv University, Tel-Aviv 69978, Israel \\ 7 Instituto de Astrofisica de Andalucia, CSIC, Aptdo. 3004, 18080 Granada, Spain \\ 8 Département de Radioastronomie ARPEGES, Observatoire de Paris, F-92195 Meudon Cedex, France \\ 9 Visiting astronomer at Calar Alto Observatory, Spain \\ 10 Visiting astronomer at Kitt Peak Observatory, U.S.A.
}

Received October 14; accepted December 22, 1999

\begin{abstract}
We present the third list with results ${ }^{1}$ of the Hamburg/SAO Survey for Emission-Line Galaxies (HSS therein, SAO - Special Astrophysical Observatory, Russia). This survey is based on the digitized objectiveprism photoplate database of the Hamburg Quasar Survey (HQS).
\end{abstract}

Here, we present new spectroscopic results of candidates which were obtained in 1998 with the $2.1 \mathrm{~m} \mathrm{KPNO}$ and the $2.2 \mathrm{~m}$ Calar Alto telescopes. All candidates are selected in the declination band $+35^{\circ}$ to $+40^{\circ}$.

The follow-up spectroscopy with the $2 \mathrm{~m}$ class telescopes confirmed 85 emission-line objects out of 113 observed candidates and allowed their quantitative spectral classification. For 80 of them, the redshifts are determined for the first time. For 5 previously known ELGs, line ratios are presented for the first time. We could classify 55 out of the 85 emission-line objects as BCG/HII galaxies or probable BCGs, 4 - as QSOs, 6 - as Seyfert galaxies, 1 - as super-association in a subluminous spiral galaxy, and 11 are low-excitation objects - either starburst nuclear (SBN), or dwarf amorphous nuclei starburst galaxies (DANS). We could not classify 8 ELGs. Further, for 8

Send offprint requests to: hopp@usm.uni-muenchen.de

* Deceased 1996 September 22.

1 Tables 2 to 6 are available only in electronic form at the CDS via anonymous ftp to cdsarc.u-strasbg.fr (130.79.128.5) or via http://cdsweb.u-strasbg.fr/Abstract.html. Figures A1 to A9 will be made available only in the electronic version at http://www.edpsciences.org more galaxies we did not detect any significant emission lines.

Key words: surveys - galaxies: fundamental parameters — galaxies: distances and redshifts — galaxies: starburst - galaxies: compact

\section{Introduction}

Objective prism surveys for emission-line galaxies (ELGs) are the main source of large samples of both AGNs and galaxies with enhanced star formation (SF) activity. Several large samples of ELGs were published since the end of the 1980s. They include the samples of the University of Michigan (UM) survey (Salzer \& MacAlpine 1988; Salzer 1989; Salzer et al. 1989) near the equator, the Tololo and Cálan-Tololo survey samples (Terlevich et al. 1991; Maza et al. 1991) and the recent Marseille Schmidt survey (Surace \& Comte 1998) of the Southern sky.

In the Northern sky, large samples of ELGs have appeared during the last decade thanks to such objective prism surveys as the First and the Second Byurakan (SBS) surveys (Markarian et al. 1983; Izotov et al. 1993a; Stepanian 1994; Pustilnik et al. 1995), the Case survey (Pesch et al. 1995; Salzer et al. 1995; Ugryumov et al. 1998), and the Heidelberg void survey (Popescu et al. 1996, 1997, 1998). All these projects employed detection of strong emission lines on blue-sensitive photoplates. 
Table 1. Journal of observations

\begin{tabular}{|c|c|c|c|c|c|c|}
\hline $\begin{array}{l}\text { Date } \\
(1) \\
\end{array}$ & $\begin{array}{c}\text { Telescope } \\
(2) \\
\end{array}$ & $\begin{array}{l}\text { Instrument } \\
(3) \\
\end{array}$ & $\begin{array}{c}\text { Grating } \\
{[\AA / \mathrm{mm}]} \\
(4)\end{array}$ & $\begin{array}{c}\text { Wavelength } \\
\text { range }[\AA] \\
(5) \\
\end{array}$ & $\begin{array}{c}\text { Dispersion } \\
{[\AA / \text { pixel }]} \\
(6) \\
\end{array}$ & $\begin{array}{c}\text { Observed } \\
\text { number } \\
(7) \\
\end{array}$ \\
\hline $22.05-25.05 .1998$ & $2.1 \mathrm{~m} \mathrm{KPNO}$ & GoldCam & 165 & $3600-7400$ & 2.7 & 69 \\
\hline $27.05-01.06 .1998$ & $2.2 \mathrm{~m} \mathrm{CAHA}$ & CAFOS & 187 & $3700-8100$ & 8.9 & 44 \\
\hline
\end{tabular}

A complementary approach was based on the search of strong $\mathrm{H} \alpha$-emission on red objective prism plates as e.g. in the Universidad Complutense de Madrid (UCM) survey (Zamorano et al. 1994; Zamorano et al. 1996; Gallego et al. 1997), and the MBC (Montreal) survey (Coziol et al. 1993, 1994).

Despite the large effort to establish the above mentioned surveys, they yielded only relatively small complete samples on the order of $10^{2}$ blue compact galaxies (BCGs hereafter, e.g. Thuan et al. 1999). This is related to the relatively low surface density of the objects in the surveys of about $0.2-0.3$ per sq deg (e.g. Popescu et al. 1997). But only complete samples of sufficient size will allow studying the distribution of the inherent physical parameters of BCGs. The experience of all these surveys can be summarized as follows. To push progress in statistical studies of low-mass galaxies with star formation bursts, a reasonably large volume has to be surveyed and the selection has to be done by well understood selection procedures. Especially for BCGs of extremely low chemical abundances, which seem to be very rare objects in the local universe after all we have learned so far, a coverage of several $10^{3}$ square degrees down to the technical limits of the surveys is essential. These limits are at magnitudes as faint as $m_{\mathrm{b}}=18-19 \mathrm{mag}$. To derive a statistically robust sample of sufficient sizes from a very large field survey, objective selection procedures for the ELGs have to be applied.

With the data described below and in Papers I and II (Ugryumov et al. 1999; Pustilnik et al. 1999) of this series, the authors pursue the goal of creating a new large sample of His galaxies, or BCGs in a zone with a total area of the order 1500 square degrees. This region will fill the gap between the zones of the SBS and the region covered by the Case survey. The SBS is situated at $\alpha=7^{\mathrm{h}} 40^{\mathrm{m}} \div 17^{\mathrm{h}} 20^{\mathrm{m}}, \delta=+49^{\circ} \div+61^{\circ}$, while the Case covers $\alpha=8^{\mathrm{h}} 00^{\mathrm{m}} \div 16^{\mathrm{h}} 20^{\mathrm{m}}, \delta=+29^{\circ} \div+38^{\circ}$. For a description of the BCGs found in these two surveys, see Izotov et al. (1993a, 1993b), Thuan et al. (1994) and Pustilnik et al. (1995) for the SBS and Salzer et al. (1995), Ugryumov (1997), Ugryumov et al. (1998) for the Case survey which is still in progress.

Thus, the new Hamburg/SAO Survey (HSS) for emission-line galaxies leads, firstly, to the creation of a new BCG/Hı galaxy sample in a large sky region with the boundaries $7^{\mathrm{h}} 20^{\mathrm{m}}$ to $17^{\mathrm{h}} 40^{\mathrm{m}}$ in right ascension and $+35^{\circ}$ to $+50^{\circ}$ in declination. Secondly, after combining the three BCG samples in the SBS, the Case and the HSS zones, a large Northern BCG sample covering about 3000 square degrees will be available. The main goal of the project is the search for emission-line galaxies (ELG) in order to create a new deep sample of blue compact/Hin galaxies (BCG) in a large area of the sky. Another important goal of this work is to search for new extremely low-metallicity galaxies.

This is the third article of a series devoted to followup spectroscopy results of HSS ELG candidates. It deals with 113 candidates selected in the band between $+35^{\circ}$ and $+40^{\circ}$ in declination which is complementary to the zone $+40^{\circ} \div+50^{\circ}$ studied in our previous papers. The basic ideas of the HSS and its selection methods of ELG candidates are described along with the first results of the follow-up spectroscopy in Ugryumov et al. (1999) (Paper I). The final selection was slightly modified to improve significantly the detection rate of ELGs in follow-up spectroscopy as described in Paper II. In short, the ELG candidate selection criteria applied are a blue or flat continuum (near $\lambda 4000 \AA$ ) and the presence of strong or moderate [OIII] $\lambda \lambda 4959,5007 \AA$ emission lines recognized on digitized prism spectra. Candidates accepted are restricted to the $B$-magnitude range $16^{\mathrm{m}}-19{ }^{\mathrm{m}} 5$.

The article is organized as follows. In Sect. 2 we give the details of the spectroscopic observations and of the data reduction. In Sect. 3 the results of the observations are presented in several tables. Along with general parameters for the emission-line galaxies and several quasars, the parameters of the strongest emission lines of the ELGs are summarized in a separate table. The information on two non-emission-line galaxies is presented as well. In Sect. 4 we briefly discuss the new data and summarize the current state of the Hamburg/SAO survey. Throughout this paper a Hubble constant $H_{0}=75 \mathrm{~km} \mathrm{~s}^{-1} \mathrm{Mpc}^{-1}$ is used.

\section{Spectral observations and data reduction}

All results presented below were obtained by observations in a snap-shot mode during two runs with the KPNO $2.1 \mathrm{~m}$ telescope and the Calar Alto $2.2 \mathrm{~m}$ telescope (see Table 1 ). 


\subsection{Observations with the KPNO $2.1 \mathrm{~m}$ telescope}

The observations were made with the GoldCam spectrograph used in conjunction with the $3 \mathrm{~K} \times 1 \mathrm{~K}$ CCD detector. We used a $2^{\prime \prime} \times 229^{\prime \prime}$ slit with the grating 09 (316 grooves $\mathrm{mm}^{-1}$ ) in its first order, and a GG 375 order separation filter. This filter cuts off all second-order contamination for wavelengths blueward of $7400 \AA$ which is the wavelength region of interest here. This instrumental setup gave a spatial scale along the slit of 0.75 pixel $^{-1}$, a scale perpendicular to the slit of $2.7 \AA$ pixel $^{-1}$, a spectral range of $3700-7500 \AA$ and a spectral resolution of $\sim 5 \AA$. These parameters permitted cover simultaneous coverage of the blue and red spectral range with all the lines of interest in a single exposure and with enough spectral resolution to separate important emission lines such as $\mathrm{H} \gamma \lambda 4340$ and [OIII] $\lambda 4363$, and $\mathrm{H} \alpha \lambda 6563$ and [NII] $\lambda 6584$. Normally, short exposures were used ( $5 \mathrm{~min}$ ) in order to detect strong emission lines, to measure redshifts and make some crude classification.

Reference spectra of an Ar-Ne-He lamp were recorded to provide a wavelength calibration. The spectrophotometric standard star Feige 34 from Massey et al. (1988) was observed for the flux calibration at least once a night. No effort was made to orient the slit along the parallactic angle, so line flux ratios could be spectrophotometrically inaccurate. The observations were complemented by dome flats, bias-, and dark frames. The seeing was about $3^{\prime \prime}$ (FWHM).

\subsection{Calar Alto 2.2 $m$ telescope observations}

Follow-up spectroscopy with this telescope was conducted as a back-up for a main program which needed photometric conditions. So, the observations presented here were obtained in non-photometric conditions and the absolute flux calibration of the data is unreliable.

The Cassegrain focal reducer CAFOS of the $2.2 \mathrm{~m}$ telescope was used with a long slit of $300^{\prime \prime} \times 3^{\prime \prime}$ and a grism of $187 \AA \mathrm{mm}^{-1}$ linear dispersion. Spectra were recorded on a $2 \mathrm{~K} \times 2 \mathrm{~K}$ Site $\mathrm{CCD}$ operated in a $2 \times 1$ binned mode (binning only along the dispersion direction), resulting in a spectral resolution of about $20 \AA$ (FWHM), and a wavelength coverage $\lambda=3700-8100 \AA$. No order separation filter was installed. The slit orientation was again not aligned with the parallactic angle to keep the duty-cycle high. The exposure times varied between 10 and 15 min depending on the object brightness. The observations were complemented by standard star flux measurements, $\mathrm{Hg}-$ He-Cd lamp exposures for wavelength calibration, dome flat-, bias-, and dark-frames. The seeing was between 1.5 and $2.5^{\prime \prime}$ (FWHM).

\subsection{Data reduction}

\subsubsection{Reduction of the KPNO $2.1 \mathrm{~m}$ telescope data}

The KPNO two-dimensional spectra were bias subtracted and flat-field corrected. We then use the $\mathrm{IRAF}^{2}$ software routines IDENTIFY, REIDENTIFY, FITCOORD, TRANSFORM to do the wavelength calibration and the correction for distortion and tilt for each frame. Then the one-dimensional spectra were extracted from each frame using the APALL routine without weighting. For all objects we extracted the brightest part of the galaxy covering a spatial size of $7^{\prime \prime}$. All extracted spectra from the same object were then co-added. Cosmic ray hits have been removed manually. To derive the instrumental response function, we have fitted the observed spectral energy distribution of the standard star Feige 34 with a high-order polynomial.

\subsubsection{Reduction of the Calar Alto $2.2 \mathrm{~m}$ telescope data}

This reduction was fully done at SAO with the standard reduction system MIDAS (Munich Image Data Analysis System, Grosbøl 1989). We applied the context LONG as follows: bias and dark subtraction, flat-fielding, cosmic-ray removal. After the wavelength mapping, a night sky 2-D background subtraction was performed. 1-D spectra were extracted by adding the consecutive CCD rows centered on the object intensity peak along the slit. Then the corrections for atmospheric extinction and flux calibration were applied. Despite of the non-photometric observing conditions, we corrected the spectra for the instrumental response with a response curve established by observations of the spectrophotometric standard star $\mathrm{BD}+33^{\circ} 2642$.

\subsubsection{Line parameter measurements}

In the final spectra, redshifts and line fluxes are measured within MIDAS, applying Gaussian fitting to the emission lines. To determine redshifts for individual galaxies, averages are taken over the prominent individual emission lines (mostly $\mathrm{H} \beta, \mathrm{H} \alpha$, [OIII] $\lambda 4959,5007 \AA$ ). The line [OII] $\lambda 3727 \AA$ is not included in the redshift determination since for most of the objects its observed wavelength is determined with significantly larger uncertainties due to the extrapolation of the linear scale below the first line of the reference spectrum (HeI $\lambda 3889 \AA$ A). However [OII] $\lambda 3727 \AA$ was used to determine the redshift in rare cases where it is the only strong emission line. The errors of the redshift in such cases can be several times larger than the typical one (compare Table 2).

\footnotetext{
${ }^{2}$ IRAF is distributed by National Optical Astronomical Observatories, which is operated by the Association of Universities for Research in Astronomy, Inc., under cooperative agreement with the National Science Foundation.
} 
To improve the accuracy of the redshift determination for the Calar Alto spectra, and further, to reduce possible small systematic shifts in the zero point of the wavelength calibration, we additionally checked the wavelengths of night sky emission lines on the 2-D spectra at the position of the object spectrum. If some measurable shift was detected it was incorporated in measurements of emission line positions.

The emission line fluxes are computed by summing up the pixel intensities inside the line region applying standard MIDAS program tools. For all spectra, the individual emission line fluxes of the $\mathrm{H} \alpha,[\mathrm{NII}] \lambda \lambda 6548,6583 \AA$ and [SII] $\lambda \lambda 6716,6731 \AA$ line blends are obtained by summing up pixel intensities over the total blend and then modeling the individual line fluxes using Gaussian fitting.

\section{Results of follow-up spectroscopy}

In total 108 new candidates and 5 known ELGs have been observed. Among them, 81 are new or confirmed emissionline galaxies, 4 are quasars (all with redshifts in the range 3.07 to 3.20 ), and 8 are galaxies without emission lines. Only 2 of the latter have good enough $\mathrm{S} / \mathrm{N}$ ratio to identify absorption features enabling measurements of their redshifts. The remaining 20 objects appeared to be either stars with characteristic absorption lines or stellar objects with featureless spectra where the signal-to-noise ratio was insufficient to identify lines.

\subsection{Emission-line galaxies}

The new emission line galaxies are listed in Table 2 containing the following information:

Column 1: The object's IAU-type name with the prefix HS. We note by asterisk objects observed at Calar Alto. Column 2: Right ascension for equinox B1950.

Column 3: Declination for equinox B1950. The coordinates were measured on direct plates of the HQS and are accurate to $\sim 2^{\prime \prime}$ (Hagen et al. 1995).

Column 4: Heliocentric velocity and its rms uncertainty in $\mathrm{km} \mathrm{s}^{-1}$

Column 5: Apparent B-magnitude obtained by calibration of the digitized photoplates with photometric standard stars (Engels et al. 1994), having an rms accuracy of $\sim 0.5$ for objects fainter than $m_{B}=16^{\mathrm{m}} 0$ (Popescu et al. 1996). Since the algorithm to calibrate the objective prism spectra is optimized for point sources the brightnesses of extended galaxies are underestimated. The resulting systematic uncertainties are expected to be as large as 2 mag (Popescu et al. 1996). For about $1 / 3$ of our objects, $B$ magnitudes are unavailable at the moment. We present for them blue magnitudes obtained from the APM database. They are marked by a "plus" before the value in the corresponding column. According to our estimate they are systematically brighter by 0.92 than the $B$-magnitudes obtained by calibration of the digitized photoplates (rms 1. 02$)$.

Column 6: Absolute B-magnitude, calculated from the apparent $B$-magnitude and the heliocentric velocity. No correction for galactic extinction is made as all objects are located at high galactic latitudes and because the corrections are significantly smaller than the uncertainties of the magnitudes.

Column \%: Preliminary spectral classification type according to the spectral data presented in this article. BCG means that the galaxy posesses a characteristic HII-region spectrum and that the luminosity is low enough. SBN and DANS are galaxies of lower excitation with a corresponding position in line ratio diagrams, as discussed in Paper I. SBN are the brighter fraction of this type. We here follow the notation of Salzer et al. (1989). Seyfert galaxies are separated mainly on diagnostic diagrams as AGN. But if their emission lines are quite narrow, they probably should be classified as Sy2. SA is a probable super-association at the rim of an edge-on nearby disc galaxy. Six objects are difficult to classify. They are coded as NON.

Column 8: One or more alternative names, according to the information from $\mathrm{NED}^{2}$.

The spectra of all emission-line galaxies are shown in Appendix A, which is available only in the electronic version of the journal.

The results of line flux measurements are given in Table 4. It contains the following information:

Column 1: The object's IAU-type name with the prefix HS. By asterisk we note the objects observed during non-photometric conditions.

Column 2: Observed flux (in $10^{-16} \mathrm{erg} \mathrm{s}^{-1} \mathrm{~cm}^{-2}$ ) of the $\mathrm{H} \beta$ line. For the few objects without an $\mathrm{H} \beta$ emission line the fluxes are given for $\mathrm{H} \alpha$ marked by a "plus". For the objects observed on Calar Alto during non-photometric conditions this parameter is unreliable and marked by $(:)$. Columns 3, 4, 5: The observed flux ratios $[\mathrm{O} \mathrm{II}] / \mathrm{H} \beta$, [OIII] $/ \mathrm{H} \beta$ and $\mathrm{H} \alpha / \mathrm{H} \beta$.

Columns 6, 7: The observed flux ratios [NII] $\lambda 6583 \AA / \mathrm{H} \alpha$, and $([\mathrm{SiI}] \lambda 6716 \AA+\lambda 6731 \AA) / \mathrm{H} \alpha$.

Columns 8, 9, 10: Equivalent widths of the lines [OII] $\lambda 3727 \AA, \mathrm{H} \beta$ and [OIII] $\lambda 5007 \AA$. For the few objects without a detected $\mathrm{H} \beta$ emission line the equivalent widths are given for $\mathrm{H} \alpha$ marked by a "plus".

Below we give notes on several individual objects:

$H S 1015+371 \%$ : In the spectrum of this object a cosmic ray hit is exactly on the line [OIII] $\lambda 4959 \AA$. This was not corrected in the figure shown in Appendix A.

$H S$ 1214+3801: This is seemingly a supergiant HII-region at the very rim of the nearby edge-on disc galaxy

\footnotetext{
${ }^{2}$ NED is operated by the Jet Propulsion Laboratory, California Institute of Technology, under contract with the National Aeronautics and Space Administration.
} 
$(\mathrm{SA}(\mathrm{s}) \mathrm{cd}) \mathrm{NGC} 4244\left(V_{\text {hel }}=224 \mathrm{~km} \mathrm{~s}^{-1}\right.$ and $\left.B_{\mathrm{T}}=10.88\right)$. At the accepted distance of NGC $4244(D=4.5 \mathrm{Mpc})$ $M_{B}$ of HS $1214+3801$ is about -11 . 8 . The difference between the systemic radial velocity of the host galaxy and HII-region is small $\left(32 \mathrm{~km} \mathrm{~s}^{-1}\right)$ and does not contradict that HS $1214+3801$ belongs to NGC 4244 . However, the velocity field of NGC 4244 near the position of the HIIregion is unkown. Both, the single-dish HI-measurements as summarized in Huchtmeier \& Richter (1989), and an estimate of the maximum rotational velocity $V_{\text {rot }} \leq$ $130 \mathrm{kms}^{-1}$ (which we obtained through the TullyFisher relation from the absolute $B$-band magnitude of NGC 4244 of $\sim-17^{\mathrm{m}} 8$ ), yield a range of expected velocity differences between the galaxian material and HS $1214+3801$ of up to +160 or $-100 \mathrm{kms}^{-1}$. But since the 2-D spectrum of HS $1214+3801$ with a total spatial extent of about $20^{\prime \prime}(\approx 0.5 \mathrm{kpc})$ shows evidence of internal motions with an amplitude of about $50 \mathrm{kms}^{-1}$ we need to consider an alternative interpretation for this object as a companion BCG. Its SF burst may be triggered due to the tidal effect from the more massive galaxy, similar to the case of HS $1717+4955$ described in Kniazev et al. (2000). To check this option one needs a detailed map of the NGC 4244 velocity field including HS 1214+3801.

$H S$ 1214+3922: This BCG was reobserved with higher $\mathrm{S} / \mathrm{N}$ ratio in order to measure the flux of the [OIII] $\lambda 4363$ line, necessary to determine unambiguously the electron temperature $T_{\mathrm{e}}([\mathrm{OIII}])$ of the HII-region and the oxygen abundance. A preliminary determination according to the procedure described by Izotov et al. (1997) shows that it has the low oxygen abundance of $\log (\mathrm{O} / \mathrm{H})+12=7.76$.

\subsection{Quasars}

In the course of our follow-up spectroscopy, four QSOs were discovered with a strong emission line in the wavelength region between $5000 \AA$ and the sensitivity break of the Kodak IIIa-J photoemulsion near $5400 \AA$. In all of them, we identified Ly $\alpha \lambda 1216$ redshifted to $z \sim 3$ as the responsible line. This strong line produces an easily visible emission peak in the digitized prism spectra even for very faint objects $\left(B \sim 19^{\mathrm{m}} 0-20^{\mathrm{m}} 0\right)$ which is hard to distinguish from low-redshift [OIII] features. Else, QSOs were not selected as candidates for follow-up spectroscopy.

The data for these four new high-redshift quasars are presented in Table 3. Finding charts and plots of their spectra can be found on the www-site of the Hamburg Quasar Survey (http://www.hs.unihamburg.de/hqs.html).

\subsection{Non-emission-line objects}

In total, for 28 candidates no (trustworthy) emission lines are detected. We divided them into three categories.

\subsubsection{Absorption-line galaxies}

For two bright non-ELG galaxies the signal-to-noise ratio of spectra was sufficient to detect absorption lines, allowing the determination of redshifts. The data are presented in Table 5 .

\subsubsection{Stellar objects}

To separate the stars among the objects missing detectable emission lines we cross-correlated a list of the most common stellar features with the observed spectra. In total, 13 objects with definite stellar spectra and redshifts close to zero were identified. Four of them are obvious $\mathrm{K}$ or $\mathrm{M}$-stars. The rest were classified roughly in categories from definite A-stars to F or G-stars, with most of them intermediate between $\mathrm{F}$ and $\mathrm{G}$. The data for these stars are presented in Table 6 .

\subsubsection{Non-classified objects}

Thirteen non emission-line objects are hard to classify at all. Their continua have too low signal-to-noise ratio to detect trustworthy absorption features, or the equivalent width of the emission lines is too small. Six of them are certainly non-stellar on DSS images, and classified as well as non-stellar in the APM database. From our spectra in the range $\approx 4000$ to $\approx 7300-8000 \AA$, we can exclude the presence of strong $\mathrm{H} \alpha$. The remaining 7 objects are indistinguishable from stellar ones, and we suggest that most of them are galactic stars. One of the galaxies - namely HS $1232+3609$, was presented after our observations in the paper by Popescu et al. (1998) as an ELG with $z=0.2529$. Our spectrum is too noisy, and we could not identify any significant emission with this redshift.

\section{Discussion}

Altogether we have observed 113 objects preselected as ELG candidates on HQS objective prism plates, of which 108 had no previous spectroscopic information. Of those 85 objects $(75 \%)$ are found to be either ELGs, or quasars. Of 81 detected ELGs, 55 were classified based on the character of their spectra and their absolute magnitudes as HII/BCGs or probable BCGs. According to their line intensity ratios, six galaxies are of the Sy type, five of them probably of type Sy2, while the continuum bump blueward to $\mathrm{H} \beta$ in the spectrum of HS $1526+3729$ suggests an identification as the Fe II emission line blend typical for Sy1 galaxies. But we caution the relatively low $\mathrm{S} / \mathrm{N}$ ratio in that part of the spectrum. One very faint object (HS 1214+3801) of absolute magnitude $M_{B}=$ -11.8 is probably a super-association in the dwarf spiral NGC 4244, or a BCG companion to this subluminous 
spiral galaxy. Eight candidates are difficult to classify. The remaining 11 ELGs are objects of lower excitation: either starburst nuclei galaxies (SBN and probable SBN) or their lower mass analogs, dwarf amorphous nuclear starburst galaxies (DANS or probable DANS). Since the main goal of the HSS is an efficient search for new BCGs, the fraction of this type among all new detected ELGs $(\sim 68 \%$, or $65 \%$ among all emission-line objects) is encouraging.

The distributions of the new HSS ELGs in the lineratio diagrams [OIII] $\lambda 5007 / \mathrm{H} \beta$ versus [NII] $\lambda 6583 / \mathrm{H} \alpha$ and [OIII] $\lambda 5007 / \mathrm{H} \beta$ versus [OII] $\lambda 3727 /[\mathrm{OIII}] \lambda 5007$ (see Baldwin et al. (1981), Veilleux \& Osterbrock (1987) for details) in general are similar to those shown in Paper I.

Compared to Paper II, we picked up significantly fewer low-luminosity ELGs $\left(M_{B} \leq-15\right)$. This is partly connected to the modest size of the telescopes used $(2 \mathrm{~m}$ versus $6 \mathrm{~m}$ in Paper II) and the range of apparent brightness of ELG candidates observed for this paper. Probably more important is that the Calar Alto observations prefered apparently bright objects (only 4 fainter than 18.5) due to the back-up status of the measurements which was prompted by a modest weather quality.

Altogether in Papers I through III, we discovered 257 new emission-line objects (14 of them QSOs), and for 35 more galaxies we got quantitative data for their emission lines. Preliminary classification of the 278 ELGs yields 206 confident or probable blue compact/low-mass Hiı galaxies. Thus a large fraction of BCGs relative to all ELGs is found ( $\sim 74 \%$ ) demonstrating the high efficiency of this survey to find galaxies with HiI-type spectra on the Hamburg Quasar Survey photoplates. A statistical analysis of this BCG sample, supplemented with galaxies from the next slices of the survey, is underway.

\section{Conclusions}

We conducted follow-up spectroscopy within the third declination slice of candidates from the Hamburg/SAO Survey for ELGs. Summarizing the results presented, the analysis of the content of various types of objects, and the discussion above, we draw the following conclusions:

- The intended methods to detect ELG candidates on the plates of the Hamburg Quasar Survey give a reasonably high detection rate of emission-line objects ( 75\%) (85 objects of 113 observed in this third part);

- Besides ELGs, we found also 4 new quasars, all with Ly $\alpha$ in the wavelength region $4950-5100 \AA$ (i.e with $3.07<z<3.2)$ near the red boundary of the IIIa-J photoplates;

- The high fraction of BCG/HII galaxies among all newly discovered ELGs (about $68 \%$ in this paper) is in line with our main goal - to pick up a deep BCG sample in the sky region under analysis.

Acknowledgements. This work was supported by the grant of the Deutsche Forschungsgemeinschaft No. 436 RUS 17/77/94.
U.A.V. is grateful to the staff of the Hamburg Observatory for their hospitality and kind assistance. Y.I.I. thanks the staff of the National Optical Astronomy Observatories for their kind hospitality. Support by the INTAS grant No. 96-0500 was crucial to proceed with the Hamburg/SAO survey declination band centered on $+37.5^{\circ}$. SAO authors appreciate the partial financial support from the Russian Foundation for Basic Research grant No. 96-02-16398 and from the Russian Center of Cosmoparticle Physics "Cosmion". The authors acknowledge the use of the NASA/IPAC Extragalactic Database (NED).

\section{References}

Baldwin J.A., Phillips M.M., Terlevich R., 1981, PASP 93, 5 Coziol R., Demers S., Pena M., Barneoud R., 1994, AJ 108, $405 \mathrm{C}$

Coziol R., Demers S., Pena M., Torres-Peimbert S., Fontain G., Wesemael F., Lamontagne R., 1993, MNRAS 261, 170

Engels D., Cordis L., Köhler T., 1994, Proc. IAU Symp. 161, MacGillivray H.T. (ed.). Kluwer, Dordrecht, p. 317

Gallego J., Zamorano J., Rego M., Vitores A.G., 1997, ApJ 475,502

Grosbøl P., 1989, Clark G. (ed.), Rev. Mod. Astron. 2, 242

Hagen H.-J., Groote D., Engels D., Reimers D., 1995, A\&AS 111,195

Huchtmeier W., Richter O., 1989, A General Catalog of HI Observations of Galaxies. New York, Springer-Verlag

Izotov Y.I., Guseva N.G., Lipovetsky V.A., et al., 1993a, Astron. Astrophys. Trans. 3, 179

Izotov Y.I., Lipovetsky V.A., Guseva N.G., 1993b, in: "The Feedback of Chemical Evolution on the Stellar Content of Galaxies", Alloin D. \& Stasinska G. (eds.), p. 127

Izotov Yu.I., Thuan T.X., Lipovetsky V.A., 1997, ApJS 108, 1 Kniazev A.Yu., Pustilnik S.A., Ugryumov A.V., Kniazeva T.F., 2000, Astron. Lett. 26, issue 2 (in press)

Markarian B.E., Lipovetsky V.A., Stepanian J.A., 1983, Afz 19,29

Massey P., Strobel K., Barnes J.V., Anderson E., 1988, ApJ 328,315

Maza J., Ruiz M.T., González L.E., Wischnjewsky M., Peña M., 1991, A\&AS 89, 389

Pesch P., Stephenson C.B., MacConnell D.J., 1995, ApJS 98, 41

Popescu C.C., Hopp U., Elsässer H., 1997, A\&A 328, 756

Popescu C.C., Hopp U., Hagen H.-J., Elsässer H., 1996, A\&AS 116,43

Popescu C.C., Hopp U., Hagen H.J., Elsässer H., 1998, A\&AS 133, 13

Pustilnik S.A., Engels D., Ugryumov A.V., Lipovetsky V.A., Hagen H.-J., Kniazev A.Y., Izotov Y.I., Richter G., 1999, A\&AS 135, 299 (Paper II)

Pustilnik S.A., Ugryumov A.V., Lipovetsky V.A., Thuan T.X., Guseva N.G., 1995, ApJ 443, 499

Salzer J.J., 1989, ApJ 347, 152

Salzer J.J., McAlpine G.M., 1988, AJ 96, 1192

Salzer J.J., MacAlpine G.M., Boroson T.A., 1989, ApJS 70, 479

Salzer J.J., Moody J.W., Rosenberg J.L., Gregory S.A., Newberry M.V., 1995, AJ 109, 2376

Stepanian J.A., 1994, Proc. IAU Symp. 161. Kluwer, Dordrecht, MacGillivray H.T. (ed.), p. 731 
Surace C., Comte G., 1998, A\&AS 133, 171

Terlevich R., Melnick J., Masegosa J., Moles M., Copetti M.V.F., 1991, A\&AS 91, 285

Thuan T.X., Izotov Y.I., Lipovetsky V.A., Pustilnik S.A., 1994, Proc. ESO/OHP Workshop "Dwarf Galaxies", Meylan \& Prugniel (eds.), p. 421

Thuan T.X., Lipovetsky V.A., Martin J.-M., Pustilnik S.A., 1999, A\&AS 139, 1

Ugryumov A.V., 1997, Ph.D. Thesis, SAO RAS
Ugryumov A.V., Engels D., Lipovetsky V.A., Hagen H.-J., Hopp U., Richter G.M., Pustilnik S.A., Kniazev A.Y., Izotov Y.I., Popescu C.C., 1999, A\&AS 135, 511 (Paper I) Ugryumov A.V., Pustilnik S.A., Lipovetsky V.A., Izotov Y.I., Richter G.M., 1998, A\&AS 131, 295

Veilleux S., Osterbrock D.E., 1987, ApJS 63, 295

Zamorano J., Gallego J.G., Rego M., Vitores A. G., Alonso O., 1996, ApJS 105, 343

Zamorano J., Rego M., Gallego J., et al., 1994, ApJS 95, 387 